\title{
ACR TI-RADS and ATA US scores are helpful for the management of thyroid nodules with indeterminate cytology
}

Thayse Lozovoy Madsen Barbosa ${ }^{1,2^{*}}$ (D), Cleo Otaviano Mesa Junior ${ }^{1,2}$, Hans Graf ${ }^{1,2}$, Teresa Cavalvanti ${ }^{3}$, Marcus Adriano Trippia ${ }^{4}$, Ricardo Torres da Silveira Ugino ${ }^{5}$, Gabriel Lucca de Oliveira ${ }^{5}$, Victor Hugo Granella ${ }^{5}$ and Gisah Amaral de Carvalho ${ }^{1,2}$

\begin{abstract}
Background: Cytologically indeterminate thyroid nodules currently present a challenge for clinical decision-making. The main aim of our study was to determine whether the classifications, American College of Radiology (ACR) TIRADS and 2015 American Thyroid Association (ATA) guidelines, in association with The Bethesda System for Reporting Thyroid Cytopathology (TBSRTC), could be used to stratify the malignancy risk of indeterminate thyroid nodules and guide their clinical management.

Methods: The institutional review board approved this retrospective study of a cohort of 140 thyroid nodules in 139 patients who were referred to ultrasound-guided fine-needle aspiration cytology (FNAC) from January 2012 to June 2016 with indeterminate cytological results (44 Bethesda III, 52 Bethesda IV and 44 Bethesda V) and in whom pre-FNAC thyroid US images and histological results after surgery were available. Each included nodule was classified by one radiologist blinded to the cytological and histological diagnoses according to the ACR TIRADS scores and the US patterns as recommended in the 2015 ATA guidelines. The risk of malignancy was estimated for Bethesda, TI-RADS scores, ATA US patterns and their combination.

Results: Of the 140 indeterminate thyroid nodules examined, 74 (52.9\%) were histologically benign. A different rate of malignancy $(p<0.001)$ among Bethesda III, IV and V was observed. The rate of malignancy increased according to the US suspicion categories $(p<0.001$ ) in both US classifications (TI-RADS and ATA). Thyroid nodules classified as Bethesda III and the lowest risk US categories (very low, low and intermediate suspicion by ATA and 2, 3 and 4a by TI-RADS) displayed a sensitivity of $95.3 \%$ for both classifications and a negative predictive value of 94.3 and $94.1 \%$, respectively. The highest risk US categories (high suspicion by ATA and $4 \mathrm{~b}, 4 \mathrm{c}$ and 5 by TI-RADS) were significantly associated with cancer (odds ratios [ORs] 14.7 and 9.8, respectively).

Conclusions: Ultrasound classifications, ACR TI-RADS and ATA guidelines, may help guide the management of indeterminate thyroid nodules, suggesting a conservative approach to nodules with low-risk US suspicion and Bethesda III, while molecular testing and surgery should be considered for nodules with high-risk US suspicion and Bethesda IV or V.
\end{abstract}

Keywords: Thyroid nodules, Indeterminate, ACR TI-RADS, American Thyroid Association (ATA) guidelines, Ultrasonography

\footnotetext{
* Correspondence: thayselozovoy@hotmail.com

'Department of Endocrinology and Metabology, Federal University of Paraná- Brazil, Av. Agostinho Leão Júnior, 285, Alto da Glória, Curitiba, PR 80.030-110, Brazil

${ }^{2}$ Department of Endocrinology, Clinical Hospital of the Federal University, Av. Agostinho Leão Júnior, 285, Alto da Glória, Curitiba, PR 80.030-110, Brazil

Full list of author information is available at the end of the article
}

(c) The Author(s). 2019 Open Access This article is distributed under the terms of the Creative Commons Attribution 4.0 International License (http://creativecommons.org/licenses/by/4.0/), which permits unrestricted use, distribution, and reproduction in any medium, provided you give appropriate credit to the original author(s) and the source, provide a link to the Creative Commons license, and indicate if changes were made. The Creative Commons Public Domain Dedication waiver (http://creativecommons.org/publicdomain/zero/1.0/) applies to the data made available in this article, unless otherwise stated. 


\section{Background}

Thyroid nodules (TNs) are very common in clinical practice, with a prevalence of up to $68 \%$ by US in the general population [1]. The challenge of the clinician is to exclude thyroid cancer, which occurs in a small subgroup of nodules $(\sim 10 \%)$ [2]. Establishing a differential diagnosis is essential to avoid unnecessary surgeries in asymptomatic benign nodules and delayed diagnosis and treatment for malignant lesions. Fine-needle aspiration cytology (FNAC) is an accurate and cost-effective tool in which benign and malignant diagnoses carry malignancy risks of approximately $<5$ and $>96 \%$, respectively [3]. Unfortunately, approximately $25 \%$ of all biopsies have indeterminate cytology [4]. The Bethesda System for Reporting Thyroid Cytopathology (TBSRTC) classifies indeterminate cytological results into 3 of 6 categories: Bethesda III - Atypia of Undetermined Significance/Follicular Lesion of Undetermined Significance (AUS/ FLUS), Bethesda IV - Follicular Neoplasm/Suspicious for a Follicular Neoplasm (FN/SFN) and Bethesda V - Suspicious for Malignancy (SM) [5]. For indeterminate TNs, molecular tests are proposed in an attempt to refine the preoperative diagnosis to reduce the rate of diagnostic surgeries [6, 7]. However, the significant cost of the molecular markers and their unavailability in all health-care centers make their use in clinical practice unfeasible.

Ultrasound (US) has a central role in the evaluation of TNs that are eligible for FNAC [8, 9]. Two of the most widely known thyroid nodule US classifications are the Thyroid Imaging Reporting and Data System (TI-RADS) and the 2015 American Thyroid Association guideline (2015 ATA) [2]. Initially, TI-RADS was reported by Horvath et al. [10], as well as a subsequent proposal by Park et al. [11] and Kwak et al. [12]. In 2017, the American College of Radiology (ACR) recommended a point system for the assessment of imaging TNs. Points are assigned based on 5 ultrasound features and the sum determines the ACR TI-RADS classification of the nodule [13]. The 2015 ATA guidelines classified the TNs into five patterns according to the combination of US features and each pattern had an estimated risk of malignancy (Table 1). According to the size and TI-RADS score or ATA pattern, the nodule was referred for FNAC or follow-up. Features that raise the suspicion for thyroid cancer are marked hypoechogenicity, microcalcifications, irregular margins, and taller-than-wide shape [12, 14-17]. The performance of 2017 ACR TI-RADS was compared with two other well-established guidelines, including 2015 ATA by Middleton et al. [18] with favorable comparison among the US reporting systems in predicting the malignancy risk.

Few studies have addressed the role of US scores in indeterminate TNs [19-24]. Gao et al. [25] suggested that US is helpful for differentiating benign and malignant
Table 1 US patterns and estimated risk of malignancy proposed by American Thyroid Association ATA, 2015

Benign - Estimated risk of malignancy $<1 \%$

US features: Purely cystic nodules (no solid component)

Very low suspicion risk - Estimated risk of malignancy $<3 \%$

US features: Spongiform or partially cystic nodules without any of US features defining low-, intermediate- or high-suspicion patterns

Low suspicion risk - Estimated risk of malignancy 5-10\%

US features: Iso- or hyperechoic solid nodule, or partially cystic nodule with eccentric solid areas, without microcalcification, irregular margins, extrathyroidal extension (ETE) or taller-than-wide shape

Intermediate suspicion risk - Estimated risk of malignancy 10-20\%

US features: Hypoechoic solid nodule with smooth margins without microcalcification, ETE or taller-than-wide shape

High suspicion risk - Estimated risk of malignancy > 70-90\%

US features: Solid hypoechoic nodule or solid hypoechoic component of a partially cystic nodule with one or more of the following features: irregular margins (microlobulated, infiltrative), microcalcification, tallerthan-wide shape, rim calcifications with small extrusive soft tissue component or ETE

Bethesda III through a meta-analysis that included 2405 nodules. The specificity was $71 \%$ and sensitivity was $66 \%$ in nodules with 3 suspicious US features. The clinical significance of TI-RADS and 2015 ATA guidelines in the subcategorization of Bethesda III (AUS and FLUS) was estimated by Baser et al. [26] and Lee et al. [24], respectively. In both studies, malignant nodules in the AUS group had a significantly higher prevalence of suspicious US features than benign nodules. He et al. investigated the diagnostic performance of TI-RADS and a new US scoring system defined according to the risk score that was calculated based on marked hypoechogenicity, taller-than-wide and absence of the halo sign in indeterminate TNs. When the two methods were analyzed (TI-RADS and the new US scoring) in combination, the performance was superior to the use of TI-RADS alone with sensitivity, specificity, positive predictive value (PPV), negative predictive value (NPV) and accuracy of 97.6, 48.5, 70.9, 94.1 and 76.2\%, respectively [27]. In this scenario, we believe that the US patterns should not only select the nodule for biopsy but also triage cytologically indeterminate TNs for US followups, molecular analysis or surgery.

The combination of 2017 ACR TI-RADS or 2015 ATA with TBSRTC and its impact on the medical approach in indeterminate TNs has not been examined extensively. Our study aims to stratify the malignancy risk of indeterminate TNs (Bethesda III, IV and V) by combining the cytology and US features correlating with the final histopathology from 139 thyroidectomized patients.

\section{Methods}

A retrospective observational study with a cohort registry of patients who underwent thyroid US, FNAC with 
indeterminate results and thyroidectomy was conducted after the Institutional Review Board Approval. Written informed patient consent was obtained for review of image and medical reports.

\section{Patients}

The study was conducted at the Clinical Hospital of the Federal University of Paraná (HC-UFPR) in association with one external private institution in an iodine-replete area in Brazil. Initially, we performed a retrospective review on the database from our institution and from one external pathology center for all thyroid FNAC between January 2012 and June 2016. TNs were included in this study if they had (a) indeterminate cytology; (b) a thyroid US image and (c) surgical resection with a histopathological result matching with the nodule's location and size analyzed on US-FNAC. Exclusion criteria were lack or absence of information on the US, FNAC or histology.

\section{Thyroid FNAC and cytological interpretation}

During the study period, 11.825 nodules were biopsied in 8.058 patients by experienced clinicians, cytopathologists or radiologists under US guidance. All nodules biopsied were classified according to the Bethesda system: 2.503 (21.1\%) nondiagnostic (Bethesda I), 7.332 (62\%) benign (Bethesda II), 1.078 (9.1\%) AUS/FLUS (Bethesda III), 250 (2.1\%) FN/SFN (Bethesda IV), 207 (1.7\%) SM (Bethesda V) and 455 (3.9\%) malignant (Bethesda VI) (Fig. 1).

\section{Histopathological results}

The pathology reports pertaining to those patients who underwent surgery were searched at both pathology centers from our institution and the external private pathology center, and each nodule included in this study was considered benign or malignant for the diagnosis of thyroid cancer as our gold standard for data analysis. The lack of final histopathology was due to the loss of clinical and outpatient follow-up and difficulty in retrieving the histological results from external pathology centers. Incidentally thyroid cancer (microcarcinoma found in another nodule) was not considered for statistical analysis. The cytologic and histopathological evaluation were reviewed by an expert pathologist.

\section{Ultrasound examinations}

Pre-FNA thyroid US examinations were retrieved from the database of our institution and four private radiology centers. In total, 140 indeterminate thyroid nodules in 139 patients (one patient had two different nodules biopsied with indeterminate results) were included in this study. US features of each enrolled nodule were reviewed and recorded in our study database by one expert radiologist who was blinded regarding the cytological and histological diagnoses. Microsoft Excel 2010 was the platform used for data collection and analysis. Information about the nodules' size and location were provided to the radiologist. From static grayscale US images recorded during the original examination, the following categories were evaluated: composition, echogenicity, shape, margins and echogenic foci. Composition was classified as spongiform, mixed or solid. None of the purely cystic nodules were included in this analysis. The nodule was considered hyper-, iso- or hypoechoic to the surrounding thyroid parenchyma or as showing marked hypoechogenicity when compared with the adjacent strap muscle. Shape was classified as wider-than-tall or tallerthan-wide (greater in the anteroposterior dimension than in the transverse dimension). Margins were classified as smooth, lobulated or irregular and if there was

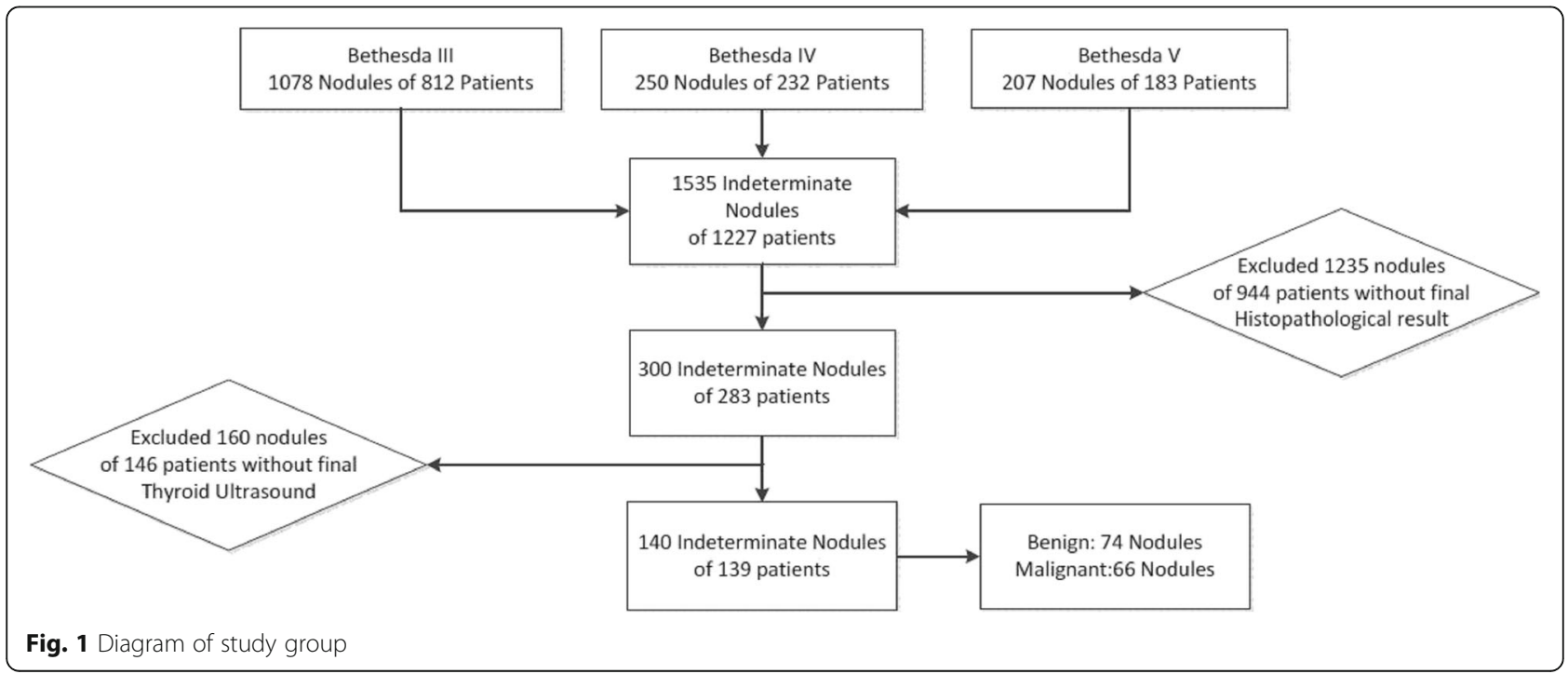


extrathyroidal extension (ETE). Echogenic foci, if present, were classified as macrocalcifications, peripheral (rim) calcifications and microcalcifications. Additionally, the presence of cervical lymphadenopathy was evaluated. US findings that were considered in favor of malignancy were hypoechoic or markedly hypoechoic echogenicity; irregular margins; presence of microcalcifications; taller-thanwide shape; evidence of ETE and the presence of lymphadenopathy.

\section{ACR TI-RADS and ATA classifications}

Based on the 2015 ATA guidelines [2], the TNs were classified according to the malignancy risk as "high", "intermediate", "low" or "very low" suspicion (Table 1). Nine nodules in our study with iso- or hypoechoic echogenicity with one suspicious US feature were classified in the high suspicion category. For the TI-RADS classification, each US feature received point(s) according to the 2017 ACR TI-RADS publication [12], being classified as TR2 (2 points); TR3 (3 points); TR4 (4-6 points) and TR5 ( $\geq 7$ points) (Table 2 ). In our study, the category TR4 was subdivided into TR4a (4 points), TR4b (5 points) and TR4c (6 points) due to its amplitude in the detection of nodules of higher or lower suspicion with scores varying from 4 to 6 points. None of the nodules were classified as "benign" by ATA or TR1 by ACR TIRADS.

\section{Statistical analysis}

The patients' ages and nodule size were described by their means and/or medians with standard deviation and/or range. Logistic regression models and Wald tests were used to evaluate factors associated with benign/malignant thyroid nodules. For statistical analysis, ACR TIRADS 2, 3 and 4a nodules, "very low", "low" and "intermediate suspicion" nodules by ATA guidelines and Bethesda III were considered favorable / negative while ACR TI-RADS 4b, 4c and 5 nodules, "high suspicion" nodules and Bethesda IV and V were considered unfavorable / positive, according to the likelihood of negative or positive results for malignancy. The level of concordance between the two ultrasound classifications (ACR TI-RADS and 2015 ATA) was estimated by the Kappa coefficient with a 95\% confidence interval (CI). Sensitivity, specificity, positive predictive value (PPV), negative predictive value (NPV) and accuracy were calculated for the combination of Bethesda and US classifications. The statistical significance was set at $p<0.05$.

Table 2 American College of Radiology Thyroid Imaging Reporting and Data System (ACR TI-RADS) Classification

\begin{tabular}{|c|c|c|c|c|}
\hline \multirow{2}{*}{\multicolumn{2}{|c|}{$\frac{\text { Ultrasound features }}{\text { Composition (choose 1) }}$}} & \multicolumn{3}{|l|}{ Description and points } \\
\hline & & \multicolumn{3}{|c|}{ Cystic or almost completely cystic - 0 points } \\
\hline & & \multicolumn{3}{|l|}{ Spongiform - 0 points } \\
\hline & & \multicolumn{3}{|c|}{ Mixed cystic and solid - 1 point } \\
\hline & & \multicolumn{3}{|c|}{ Solid or almost completely solid - 2 points } \\
\hline \multirow{4}{*}{\multicolumn{2}{|c|}{ Echogenicity (choose 1) }} & \multicolumn{3}{|l|}{ Anechoic -0 points } \\
\hline & & \multicolumn{3}{|c|}{ Hyperechoic or isoechoic - 1 point } \\
\hline & & \multicolumn{3}{|l|}{ Hypoechoic -2 points } \\
\hline & & \multicolumn{3}{|c|}{ Very hypoechoic -3 points } \\
\hline \multirow{2}{*}{\multicolumn{2}{|c|}{ Shape (choose 1) }} & \multicolumn{3}{|c|}{ Wider-than-tall - 0 points } \\
\hline & & \multicolumn{3}{|c|}{ Taller-than-wide -3 points } \\
\hline \multirow{4}{*}{\multicolumn{2}{|c|}{ Margins (choose 1) }} & \multicolumn{3}{|l|}{ Smooth - 0 points } \\
\hline & & \multicolumn{3}{|l|}{ III-defined - 0 points } \\
\hline & & \multicolumn{3}{|c|}{ Lobulated or irregular -2 points } \\
\hline & & \multicolumn{3}{|c|}{ Extra-thyroidal extension - 3 points } \\
\hline \multicolumn{2}{|l|}{ Echogenic foci } & \multicolumn{3}{|c|}{ None or large comet-tail artifacts - 0 points } \\
\hline \multirow{3}{*}{\multicolumn{2}{|c|}{ (choose all that apply) }} & \multicolumn{3}{|c|}{ Macrocalcifications - 1 point } \\
\hline & & \multicolumn{3}{|c|}{ Peripheral (rim) calcifications - 2 points } \\
\hline & & \multicolumn{3}{|c|}{ Punctate echogenic foci -3 points } \\
\hline \multicolumn{5}{|c|}{ TI-RADS Category (sum of points) } \\
\hline $\begin{array}{l}\text { TR1 (0 points) } \\
\text { Benign }\end{array}$ & $\begin{array}{l}\text { TR2 (2 points) Not } \\
\text { Suspicious }\end{array}$ & $\begin{array}{l}\text { TR3 ( } 3 \text { points) Mildly } \\
\text { suspicious }\end{array}$ & $\begin{array}{l}\text { TR4 ( } 4-6 \text { points) Moderately } \\
\text { suspicious } \\
\text { TR4a }-4 \text { points } \\
\text { TR4b }-5 \text { points } \\
\text { TR4c }-6 \text { points }\end{array}$ & $\begin{array}{l}\text { TR5 ( } \geq 7 \text { points) Highly } \\
\text { suspicious }\end{array}$ \\
\hline
\end{tabular}


Statistical software (IBM SPSS Statistics v.20.0. Armonk, NY: IBM Corp) were used to conduct the data analysis.

\section{Results}

The cohort comprised 44 (31.4\%) Bethesda III, 52 (37.1\%) Bethesda IV and 44 (31.4\%) Bethesda V TNs identified in 139 patients (118 females), with a mean age of $49 \pm 13$ years, who had undergone thyroidectomy. Seventy-four $(52.9 \%)$ of the 140 nodules were histologically diagnosed as benign (42 cases of nodular hyperplasia, 31 cases of follicular adenomas and 1 case of noninvasive follicular thyroid neoplasm with papillarylike features [NIFTP]). The $66(47.1 \%)$ nodules that were malignant included 44 papillary thyroid cancers (PTC), 12 follicular-variant papillary thyroid cancers (FVPTC), 1 Warthin-like papillary carcinoma of the thyroid, 4 medullary thyroid cancers, 4 follicular thyroid carcinomas and 1 poorly differentiated thyroid carcinoma. The overall distribution in the TI-RADS and ATA categories was as follows: 5 TR2 (3.6\%), 43 TR3 (30.7\%), 32 TR4a (22.9\%), 9 TR4b (6.4\%), 23 TR4c (16.4\%), 28 TR5 (20.0\%), 5 "very low" (3.6\%), 43 "low" (30.7\%), 36 "intermediate" (25.7\%) and 56 "high" (40.0\%) suspicion nodules.

We initially evaluated the role of the patient's age at diagnosis, sex, size of the lesion and US features in predicting malignancy. No significant association was observed for age at diagnosis, sex, or composition between the benign and malignant lesions (Table 3). However, the size of the lesion $(p=0.049)$, (marked) hypoechogenicity $(p=0.012)$, taller-than-wide nodule shape $(p=$ $0.001)$, irregular margins $(p<0.001)$, and microcalcification $(\mathrm{p}<0,001)$ were associated with nodule malignancy (Table 3).

The distribution of carcinomas among Bethesda categories III, IV and V was 15.9, 40.4 and 86.4\%, respectively $(\mathrm{p}<0.001)$ (Table 4$)$. We next evaluated whether the US classifications are associated with lesion malignancy and found a positive correlation between the TIRADS score and ATA guideline patterns, individually or pooled, and risk of malignancy. The rate of malignancy increased according to the suspicion on US $(\mathrm{p}<0.001)$ (Table 4).

The diagnostic performance of ACR TI-RADS when considering category TR2/3/4a as negative and TR4b/4c/ 5 as positive was as follows: sensitivity, $69.7 \%$; specificity, 81.1\%; positive predictive value (PPV), 76.7\%; negative predictive value (NPV), $75.0 \%$ and accuracy, $75.7 \%$. The diagnostic performance of 2015 ATA when considering "very low", "low", or "intermediate" as negative and "high" as positive was as follows: sensitivity, $69.7 \%$; specificity, 86.5\%; PPV, 82.1\%; NPV, 76.2\% and accuracy, $78.6 \%$. The kappa coefficient was estimated at 0.91 with a $95 \%$ confidence interval of $k, 0.84-0.98$, which was considered as 'substantial' agreement between TI-RADS and ATA.

In an attempt to stratify indeterminate lesions according to the risk of malignancy, we combined the cytology with 2017 ACR TI-RADS and 2015 ATA guidelines. The distribution of malignancy of the ACR TI-RADS score and 2015 ATA US patterns restricted to Bethesda III versus Bethesda IV and Bethesda V is shown in Table 5. Of the patients classified as Bethesda III and presenting a favorable ultrasound appearance (TI-RADS 2, 3 or $4 \mathrm{a} /$ ATA very low, low or intermediate), only 5.9 and $5.7 \%$ (TI-RADS and ATA, respectively) had malignant histological results. In contrast, 81.6 and $87.2 \%$ (TI-RADS and ATA, respectively) classified as Bethesda IV or V, which presented an ultrasound considered unfavorable (TI-RADS 4b, 4c and 5 / ATA high suspicion) had malignant histological results. In the discrepant groups, that is, favorable cytology (Bethesda III) and unfavorable ultrasound or unfavorable cytology (Bethesda IV or V) and favorable ultrasound the risk of malignancy was intermediate (Table 5).

To verify the diagnostic performance in the correlation between TBSRTC with ACR TI-RADS and 2015 ATA in predicting malignant anatomopathological (AP) results we calculated the parameters of sensitivity, specificity, PPV, NPV and accuracy using Bethesda III and 2/3/4a by TI-RADS or "very low", "low", "intermediate" by ATA as favorable / negative and Bethesda IV or V and $4 \mathrm{~b} / 4 \mathrm{c} /$ 5 by TI-RADS or "high" by ATA as unfavorable / positive. For correlation between Bethesda and ACR TIRADS, the parameters were as follows: sensitivity, $95.3 \%$ (89.1-100\%); specificity, 78\% (65.4-90.7\%); PPV, 82\% (71.4-92.6\%); NPV, 94.1\% (86.2-102\%) and accuracy, 86.9\% (79.7-94.1\%). For correlation between Bethesda and 2015 ATA, the parameters were as follows: sensitivity, 95.3\% (89.1-100\%); specificity, 84.6\% (73.3-95.9\%); PPV, 87.2\% (77.7-96.8\%); NPV, 94.3\% (86.6-102\%) and accuracy, 90.2\% (83.8-96.7\%).

The percentage of malignancy in the final AP was estimated according to the US classifications (ACR TIRADS [2 / 3 / 4a; 4b / 4c / 5] and 2015 ATA ["very low", "low", "intermediate", or "high"]) and Bethesda system (III; IV/V) by logistic regression model and Wald test statistical analysis. The lowest US suspicion categories (TR2/3/4a and "very low", "low", or "indeterminate") in Bethesda III had the highest likelihood of benignity, while the likelihood of malignancy in the final AP result increased progressively according to the increase in US suspicion and the Bethesda diagnostic categories (Table 6).

\section{Discussion}

The differentiation of benign and malignant lesions among cytologically indeterminate TNs has represented 
Table 3 Association of clinical and ultrasound (US) features with final histopathological result of 140 nodules with indeterminate diagnosis

\begin{tabular}{|c|c|c|c|c|}
\hline \multirow[b]{2}{*}{ Clinical and US features } & \multicolumn{4}{|c|}{ Final histopathological results } \\
\hline & Benign $(n=74)$ & Malignant $(n=66)$ & $P^{*}$ value & OR (CI 95\%) \\
\hline \multicolumn{5}{|l|}{ Age (yr) } \\
\hline Mean & $49,6 \pm 14,0(18-84)$ & $48,6 \pm 13,3(25-77)$ & 0.663 & $0.99(0.97-1.02)$ \\
\hline \multicolumn{5}{|l|}{ Gender } \\
\hline Female & $64(54.2 \%)$ & $54(45.8 \%)$ & & \\
\hline Male & $9(42.9 \%)$ & $12(57.1 \%)$ & 0.339 & $1.58(0.61-4.07)$ \\
\hline Size $(\mathrm{cm})$ & $2.0(0.72-8.33)$ & $1.43(0.54-7.57)$ & 0.049 & $0.58(0.33-0.99)$ \\
\hline \multicolumn{5}{|l|}{ Composition } \\
\hline Solid (ref) & $67(52.3 \%)$ & $61(47.7 \%)$ & & \\
\hline Mixed & $4(57.1 \%)$ & $3(42.9 \%)$ & 0.738 & $1.37(0.22-8.59)$ \\
\hline Spongiform & $3(60 \%)$ & $2(40 \%)$ & 0.921 & $1.13(0.11-11.8)$ \\
\hline \multicolumn{5}{|l|}{ Echogenicity } \\
\hline Isoechoic (ref) & $10(71.4 \%)$ & $4(28.6 \%)$ & & \\
\hline Hyperechoic & $28(65.1 \%)$ & 15 (34.9\%) & 0,664 & $1.34(0.35-5.06)$ \\
\hline Hypoechoic & $25(53.2 \%)$ & $22(46.8 \%)$ & 0,232 & $2.20(0.60-8.12)$ \\
\hline Markedly hypoechoic & $11(30.6 \%)$ & $25(69.4 \%)$ & 0,012 & $5.68(1.44-22.4)$ \\
\hline \multicolumn{5}{|l|}{ Shape } \\
\hline Wider-than-tall (ref) & $72(60.0 \%)$ & $48(40.0 \%)$ & & \\
\hline Taller-than-wide & $2(10.0 \%)$ & $18(90.0 \%)$ & 0.001 & $13.5(2.94-61.9)$ \\
\hline \multicolumn{5}{|l|}{ Margins } \\
\hline Regular & $66(69.5 \%)$ & $29(30.5 \%)$ & & \\
\hline Lob/irregular & $8(17.8 \%)$ & $37(82.2 \%)$ & $<0.001$ & $10.5(4.32-25.6)$ \\
\hline \multicolumn{5}{|l|}{ Echogenic foci } \\
\hline Absent & $70(60.9 \%)$ & $45(39.1 \%)$ & - & \\
\hline Macrocalcifications & $0(0 \%)$ & $3(100 \%)$ & & \\
\hline Peripheral calcification & $1(50 \%)$ & $1(50 \%)$ & & \\
\hline Microcalcifications & $3(15 \%)$ & $17(85 \%)$ & & - \\
\hline \multicolumn{5}{|l|}{ Echogenic foci } \\
\hline Yes & $4(16.0 \%)$ & $21(84.0 \%)$ & & \\
\hline No & $70(60.9 \%)$ & $45(39.1 \%)$ & $<0.001$ & $8.17(2.60-25.7)$ \\
\hline
\end{tabular}

Results presented as mean \pm standard deviation (minimum-maximum) or median (minimum-maximum); frequency (percent)

*Logistic regression models and Wald test; $p<0.05$

$O R$ odd ratios, $\mathrm{Cl}$ confidence interval

a challenge in clinical practice. As the overall rate of malignancy is low in indeterminate TNs, a high rate of benignancy is expected after surgery and a tool that helps to rule out thyroid cancer preoperatively might reduce the number of diagnostic surgeries and, hence, the risks involved in this procedure [28]. According to the results of our study, the combination of US classifications, ACR TI-RADS and ATA, with the Bethesda system is useful for detecting benign lesions in Bethesda III nodules and malignant lesions in Bethesda IV/V nodules. Both US classifications were effective in ruling out malignancy in Bethesda III TNs with low-risk US categories: "very low", "low" and "intermediate" - ATA and TR2, TR3 and
TR4a - ACR TI-RADS (NPV 94.1\% from ACR TI-RADS and $94.3 \%$ from ATA) while indeterminate Bethesda IV/ V TNs with high-risk US categories: "high" - ATA and TR4b, TR4c and TR5 - TI-RADS had the highest risk of malignancy (88.2 and $83.5 \%$, respectively). The NPV obtained in the Bethesda III category was quite similar to the results obtained using molecular tests $[6,7,29,30]$, reinforcing the conclusion that US classification may be helpful for decision-making in favor of a conservative approach and follow-up of nodules in this category.

Several studies in recent years have suggested the use of US patterns to stratify the risk of malignancy of indeterminate TNs [19-23, 25, 31, 32]. According to the 
Table 4 Malignancy rate according to TBSRTC, ACR TI-RADS score and 2015 ATA pattern in 140 indeterminate thyroid nodules

\begin{tabular}{|c|c|c|c|c|c|c|}
\hline \multirow[t]{2}{*}{ Classification } & \multirow[b]{2}{*}{ Number of cases } & \multicolumn{2}{|l|}{ Final Diagnosis } & \multirow[b]{2}{*}{ Risk of malignancy (\%) } & \multirow[b]{2}{*}{$P^{*}$ value } & \multirow[b]{2}{*}{$\mathrm{OR}(\mathrm{Cl} 95 \%)$} \\
\hline & & Benign $(n=74)$ & Malignant $(n=66)$ & & & \\
\hline \multicolumn{7}{|l|}{ TBSRTC } \\
\hline Bethesda III & 44 & 37 & 7 & $15.9 \%$ & & \\
\hline Bethesda IV & 52 & 31 & 21 & $40.4 \%$ & & \\
\hline Bethesda V & 44 & 6 & 38 & $86.4 \%$ & $<0.001$ & \\
\hline \multicolumn{7}{|l|}{ TBSRTC groups } \\
\hline III & 44 & 37 & 7 & $15.9 \%$ & & \\
\hline IVN & 96 & 37 & 59 & $61.5 \%$ & $<0,001$ & $8.43(3.37-21.1)$ \\
\hline \multicolumn{7}{|l|}{ ACR TI-RADS } \\
\hline TR2 & 5 & 4 & 1 & $20.0 \%$ & & \\
\hline TR3 & 43 & 33 & 10 & $23.3 \%$ & & \\
\hline TR4a & 32 & 23 & 9 & $28.1 \%$ & & \\
\hline TR4b & 9 & 4 & 5 & $55.6 \%$ & & \\
\hline TR4C & 23 & 8 & 15 & $65.2 \%$ & & \\
\hline TR5 & 28 & 2 & 26 & $92.9 \%$ & $<0.001$ & \\
\hline \multicolumn{7}{|l|}{ ACR TI-RADS groups } \\
\hline TR2/TR3/TR4a & 80 & 60 & 20 & $25.0 \%$ & & \\
\hline TR4b/TR4c/TR5 & 60 & 14 & 46 & $76.7 \%$ & $<0.001$ & $9.86(4.46-21.8)$ \\
\hline \multicolumn{7}{|l|}{2015 ATA patterns } \\
\hline Very low & 5 & 4 & 1 & $20.0 \%$ & & \\
\hline Low & 43 & 33 & 10 & $23.3 \%$ & & \\
\hline Intermediate & 36 & 27 & 9 & $25.0 \%$ & & \\
\hline High & 56 & 10 & 46 & $82.1 \%$ & $<0.001$ & \\
\hline \multicolumn{7}{|l|}{2015 ATA groups } \\
\hline Very low / low / intermediate & 84 & 64 & 20 & $23.8 \%$ & & \\
\hline High & 56 & 10 & 46 & $82.1 \%$ & $<0.001$ & $14.7(6.24-34.7)$ \\
\hline
\end{tabular}

*Logistic regression models and Wald test; $p<0.05$

Abbreviations: ACR TI-RADS American College of Radiology Thyroid Imaging Reporting and Data System, ATA American Thyroid Association, Cl confidence interval, OR odd ratios, TBSRTC The Bethesda System for Reporting Thyroid Cytopathology

Table 5 Distribution of malignancy of ACR TI-RADS score and 2015 ATA US patterns for Bethesda III vs IV and V categories in 140 indeterminate thyroid nodules

\begin{tabular}{|c|c|c|c|c|c|c|c|}
\hline \multirow[t]{2}{*}{ TBSRTC } & & \multicolumn{3}{|c|}{2017 ACR TI-RADS score } & \multicolumn{3}{|l|}{2015 ATA US patterns } \\
\hline & & TR2 / TR3 / TR4a & TR4b / TR4c / TR5 & Total & Very low / Low / Intermediate & High & Total \\
\hline \multicolumn{8}{|l|}{ Bethesda III } \\
\hline \multirow[t]{4}{*}{ Histological Result } & B & 32 & 5 & 37 & 33 & 4 & 37 \\
\hline & & $94.1 \%$ & $50.0 \%$ & & $94.3 \%$ & $45.5 \%$ & \\
\hline & M & 2 & 5 & 7 & 2 & 5 & 7 \\
\hline & & $5.9 \%$ & $50.0 \%$ & & $5.7 \%$ & $55.5 \%$ & \\
\hline \multicolumn{2}{|l|}{ Total } & 34 & 10 & 44 & 35 & 9 & 44 \\
\hline \multicolumn{8}{|c|}{ Bethesda IV and Bethesda V } \\
\hline \multirow[t]{4}{*}{ Histological Result } & B & 28 & 9 & 37 & 31 & 6 & 37 \\
\hline & & $40.4 \%$ & $18.4 \%$ & & $63.3 \%$ & $12.8 \%$ & \\
\hline & M & 19 & 40 & 59 & 18 & 41 & 59 \\
\hline & & $59.6 \%$ & $81.6 \%$ & & $36.7 \%$ & $87.2 \%$ & \\
\hline Total & & 47 & 49 & 96 & 49 & 47 & 96 \\
\hline
\end{tabular}

Histological Result: $B$ benign and $M$ malignant 
Table 6 Percentage of malignant anatomopathology (AP) combining Bethesda with ATA and ACR TI-RADS in 140 indeterminate thyroid nodules

\begin{tabular}{lll}
\hline ATA & Bethesda & Percentage of malignant AP \\
\hline Very low / low / intermediate & III & $7.00 \%$ \\
Very low / low / intermediate & IVN & $35.80 \%$ \\
High & III & $50.40 \%$ \\
High & IVN & $88.20 \%$ \\
ACR TI-RADS & & \\
2/3/4a & III & $8.10 \%$ \\
2/3/4a & IVN & $37.50 \%$ \\
4b/4c/5 & III & $42.50 \%$ \\
4b/4C/5 & IVN & $83.50 \%$ \\
\hline
\end{tabular}

results of our study, Grani et al. analyzed the accuracy of TI-RADS and ATA to stratify malignancy risk in 49 indeterminate TNs, concluding that nodules classified as TI-RADS 3 or very low suspicion could be conducted conservatively (NPV 100\% when the estimated risk of malignancy for the test positivity was set at 3\%). The PPV for intermediate and high suspicion of ATA and TI-RADS 4c was 63 and 71\%, respectively [19]. Maia et al. studied the combination of the TI-RADS score with the Bethesda system to stratify malignancy risk in 136 indeterminate TNs, showing high sensitivity (80\%) and NPV (90\%) in nodules classified as Bethesda III and TI-RADS 3 and 4a scores, implying a conservative approach in these cases. In contrast, nodules scored as TIRADS $4 \mathrm{~b}$ and 5 with Bethesda IV and V presented with a higher risk of malignancy (75 and $76.9 \%$, respectively) [20]. Rocha et al. prospectively analyzed 137 patients with 143 indeterminate TNs (Bethesda III and IV) who were referred for surgery. Considering noninvasive follicular thyroid neoplasms with papillary-like nuclear features (NIFTP) and tumors of uncertain malignant potential (TUMP) as benign, the rate of malignancy was $72,22.4,4.3,0$, and $15.4 \%$ for nodules with high suspicion, intermediate suspicion, low suspicion, very low suspicion, and undefined ultrasonographic pattern, respectively [33]. Most of the studies used one of the TIRADS classifications, but few used the ACR TI-RADS. Few studies analyzed the 2015 ATA guidelines in indeterminate TNs, and the majority used small cohorts with conflicting results [19, 21, 24, 34].

Cytologically indeterminate nodules had a malignancy risk implied in each category as follows: $10-30 \%$ in Bethesda III, $25-40 \%$ in Bethesda IV and $50-75 \%$ in Bethesda $\mathrm{V}[3,5]$. The percentage of each Bethesda category (III, IV and V) from the total of thyroid FNACs performed in the period of study was consistent with the literature $[9,35]$, presenting a chance of $13 \%$ of a thyroid FNAC having an indeterminate result. Even Bethesda III, which has wide variability in its use due to the difficulty of defining specific criteria, presents within the limit of interpretation to $7-10 \%$ of all FNACs [5]. Despite the large number of losses we had from the initial group of patients as a result of the lack of US examination and histological results, we believe that our sample was representative since the malignancy risk estimated in each Bethesda category was consistent with the literature.

In relation to the US classifications (TI-RADS and ATA), the estimated risk of malignancy of each US category was comparable to studies that specifically analyzed indeterminate TNs, since these nodules presented with a risk of malignancy higher than that found in the nodules in general (40\% versus 5 to $10 \%$ ). Chaigneau et al. analyzed 602 indeterminate TNs classified as TIRADS score 3, 4a, 4b and 5 with the following malignancy risks: $20.5,29,63.4$ and $100 \%$, respectively [36]. For ATA US patterns, Tang et al. classified 49 indeterminate TNs according to 2015 ATA guidelines with the following malignancy risk: "very low" (12\%), "low" (17\%), "intermediate" (21\%) and "high" (100\%) [34]. Among nodules classified by ATA in our study, the malignancy risks were consistent with the literature for "low suspicion" (23.3\%), "intermediate" (25\%) and "high suspicion" $(82.1 \%)$ patterns; however, the malignancy risk for "very low" $(20.0 \%)$ was higher than that found in the literature. For thyroid nodules classified by ACR TI-RADS, the malignancy risk for each score was similar to the study previously discussed: TR3 (23.3\%), TR4 (the mean of the three categories - TR4a, TR4b and TR4c: 49.6\%) and TR5 (92.9\%). Due to the variability of cancer risk observed in our study for TR4 score $(4 \mathrm{a}-28.1 \%$; $4 \mathrm{~b}$ $55.6 \%$ and $4 \mathrm{c}-65.2 \%$ ), we subdivided this category because we observed that TR4a had a behavior more similar to that of low risk, while TR4b and 4c had a behavior similar to that of high risk. Among the 5 nodules classified as "very low suspicion" and TR2 in our study, only one nodule demonstrated malignant histology, but in this case, the Bethesda category was SM, which is a category of higher cancer risk. It is noteworthy that malignancy rates increased proportionally to the risk of cancer in each category. Interestingly, from nine nodules with iso- or hypoechoic echogenicity that presented with one high suspicion US feature, such as microcalcifications, irregular margins or taller-than wide shape and were classified as having a "high" suspicion of malignancy in our study, eight ultimately had a malignant histology. Valderrabano et al. evaluated 463 indeterminate TNs (AUS/FLUS and FN/SFN) and concluded that there were no differences in the prevalence of malignancy between nodules with "low" (iso/hyperechoic) or "intermediate" (hypoechoic) suspicion patterns, concluding that hypoechogenicity alone did not seem to improve the risk stratification of indeterminate TNs [37]. In 
contrast, any suspicious US feature significantly increases the risk of malignancy of indeterminate TNs. In the same way, we identified in our study that the intermediate suspicious nodules of ATA had a behavior more similar to those of low risk than high risk.

The diagnostic performance of TI-RADS and ATA, in our study, was evaluated by the sensitivity, specificity, PPV, NPV and accuracy for each US classification and the comparison between the two US systems by the calculation of the Kappa concordance coefficient, which showed 'substantial' agreement between 2017 ACR TIRADS and 2015 ATA guidelines, being equivalent to the evaluation of the cytologically indeterminate TNs by both classifications in clinical practice. The malignancy risk progressively rises among the ACR TI-RADS categories whereas in 2015 ATA there is only one important difference between the intermediate and the high suspicion (the very low, low and intermediate suspicions behave very similarly). To the best of our knowledge, our study is the first to evaluate and compare the performance of the ACR TI-RADS and the 2015 ATA guidelines exclusively on indeterminate TNs.

Despite the many US classifications that have been investigated [13,18, 38-42], surgery is still often necessary for the diagnosis. Some US features are described as suspicious for several studies [12, 14-17], such as nodule hypoechogenicity, irregular margins, microcalcifications and taller-than-wide shape, although the diagnostic accuracy of individual US features is insufficient for surgical decision-making [43]. The analysis of these characteristics in our study is consistent with the above studies. Although the reinterpretation of the US is not recommended for the decision-making after indeterminate cytology [43], the results of our study suggested that US features may be considered to guide the clinical management of indeterminate TNs.

There were several limitations in our study. The main limitation was the loss bias of the sample, noted once we searched for patients with analyzable ultrasound examinations, and had significant problems in the final studied group due to the difficulty of retrieving thyroid US images and histological results. Despite this setback, we believe that our sample was representative once the prevalence of malignancy in each category of Bethesda (III, IV and V) is similar to the literature. In addition, the high NPV in Bethesda III with low-risk US categories may be relied upon in a service where the risk of malignancy is comparable with our study. If the service has higher risk of malignancy in this category, the correlation may not be reliable. Finally, there was a lack of information about some clinical parameters known to be associated with thyroid cancer, such as thyroid autoimmunity and TSH level, which deserve to be tested to evaluate their impact on the performance of the US.

\section{Conclusions}

In conclusion, the ACR TI-RADS score and the 2015 ATA US patterns can refine the malignancy risk assessment, suggesting a conservative approach for indeterminate Bethesda III TNs with low-risk US categories ("very low", "low" and "intermediate" and TR2, TR3 and TR4a), surgical approach for Bethesda IV and V with high-risk US categories ("high" and TR4b, TR4c and TR5) and molecular testing or diagnostic surgery for Bethesda III with high-risk US features or Bethesda IV and $\mathrm{V}$ with low-risk US features.

\section{Abbreviations \\ ACR: American College of Radiology; AP: Anatomopathological; ATA: American Thyroid Association; AUS/FLUS: Atypia of undetermined significance/follicular lesion of undetermined significance; FN/FSN: Follicular neoplasm/suspicious for a follicular neoplasm; FNAC: Fine-needle aspiration cytology; NPV: Negative predictive value; PPV: Positive Predictive Value; \\ SEMPR: Serviço de Endocrinologia e Metabologia do Paraná; SM: Suspicious for malignancy; TBSRTC: The Bethesda System for Reporting Thyroid \\ Cytopathology; TI-RADS: Thyroid imaging reporting and data system; \\ TNs: Thyroid nodules; US: Ultrasound}

\section{Acknowledgments}

The authors are grateful to the private radiology centers of Curitiba (CEDAV, CETAC, DAPI and IDEPI) and the pathology service (CITOLAB) by the contribution with this study through ultrasound, cytology and histological information.

\section{Authors' contributions}

TLMB searched all the database with the help of GLO, RTSU and VHG. HG collaborated with retrieving of the thyroid images. TLMB was a major contributor in writing the manuscript with the orientation and collaboration of the COM-J and GAC. TCC analysed and interpret the patient cytological and anatomopathological data. MAT analysed and classified all thyroid images accordingly to ACR TI-RADS and ATA guidelines. All authors read and approved the final manuscript.

\section{Funding}

This research did not receive any specific grant from any funding agency in the public, commercial or not-for-profit sector.

\section{Availability of data and materials}

The datasets used and/or analysed during the current study are available from the corresponding author on reasonable request

\section{Ethics approval and consent to participate}

This retrospective observational study was approved in June 10,2015 by the following ethical review board: Comitê de Ética em Pesquisa em Seres

Humanos do Hospital de Clínicas da Universidade Federal do Paraná (CEP HC) UFPR), with the following identification number: CAAE 45277115.3.0000.0096. Written informed patient consent was obtained for review of image and medical reports.

Consent for publication

Not applicable.

\section{Competing interests}

The authors declare that they have no competing interests.

\section{Author details}

${ }^{1}$ Department of Endocrinology and Metabology, Federal University of Paraná- Brazil, Av. Agostinho Leão Júnior, 285, Alto da Glória, Curitiba, PR 80.030-110, Brazil. '2Department of Endocrinology, Clinical Hospital of the Federal University, Av. Agostinho Leão Júnior, 285, Alto da Glória, Curitiba, PR 80.030-110, Brazil. '3Department of Pathology, Clinical Hospital of the Federal University of Paraná, Rua General Carneiro, 181, Alto da Glória, Curitiba, PR 80.060-900, Brazil. ${ }^{4}$ Radiology, Rua Marechal Deodoro, 503, Curitiba, PR, Brazil. ${ }^{5}$ Medical School, Federal University of Paraná, Curitiba, Brazil. 
Received: 26 June 2019 Accepted: 16 September 2019 Published online: 29 October 2019

\section{References}

1. Guth S, Theune U, Aberle J, Galach A, Bamberger CM. Very high prevalence of thyroid nodules detected by high frequency $(13 \mathrm{MHz})$ ultrasound examination. Eur J Clin Investig. 2009;39(8):699-706.

2. Haugen BR, Alexander EK, Bible KC, Doherty GM, Mandel SJ, Nikiforov YE, et al. 2015 American Thyroid Association management guidelines for adult patients with thyroid nodules and differentiated thyroid Cancer: the American Thyroid Association guidelines task force on thyroid nodules and differentiated thyroid Cancer. Thyroid. 2016;26(1):1-133 Available at: http:// online.liebertpub.com/doi/10.1089/thy.2015.0020.

3. Cibas ES, Ali SZ. The 2017 Bethesda system for reporting thyroid cytopathology. Thyroid. 2017;27(11):1341-6.

4. Valderrabano P, Mclver B. Evaluation and Management of Indeterminate Thyroid Nodules. Cancer Control. 2017;24(5):107327481772923 Available at: http://journals.sagepub.com/doi/10.1177/1073274817729231.

5. Cibas ES, Ali SZ. The Bethesda System for Reporting Thyroid Cytopathology Definitions, Criteria and Explanatory NotesVol. 2, Springer. 2018. 1-236 p. Available at: http://linkinghub.elsevier.com/retrieve/pii/S0012160616307096. Accessed 3 Nov 2017

6. Nikiforov YE, Ohori NP, Hodak SP, Carty SE, LeBeau SO, Ferris RL, et al. Impact of mutational testing on the diagnosis and management of patients with cytologically indeterminate thyroid nodules: a prospective analysis of 1056 FNA samples. J Clin Endocrinol Metab. 2011:96(11):3390-7.

7. Alexander EK, Kennedy GC, Baloch ZW, Cibas ES, Chudova D, Diggans J, et al. Preoperative diagnosis of benign thyroid nodules with indeterminate cytology. N Engl J Med. 2012;367(8):705-15 Available at: http://www.nejm. org/doi/10.1056/NEJMoa1203208.

8. Brito JP, Gionfriddo MR, Al Nofal A, Boehmer KR, Leppin AL, Reading C, et al. The accuracy of thyroid nodule ultrasound to predict thyroid Cancer: systematic review and meta-analysis. J Clin Endocrinol Metab. 2014;99(4): 1253-63 Available at: http://academic.oup.com/jcem/article-lookup/doi/1 0.1210/jc.2013-2928.

9. Durante C, Grani G, Lamartina L, Filetti S, Mandel SJ, Cooper DS. The diagnosis and Management of Thyroid Nodules. JAMA. 2018;319(9):914-24 Available at: http://jama.jamanetwork.com/article.aspx?doi=10.1001/jama.201 8.0898

10. Horvath E, Majlis S, Rossi R, Franco C, Niedmann JP, Castro A, et al. An ultrasonogram reporting system for thyroid nodules stratifying cancer risk for clinical management. J Clin Endocrinol Metab. 2009;94(5):1748-51.

11. Park J-Y, Lee HJ, Jang HW, Kim HK, Yi JH, Lee W, et al. A proposal for a thyroid imaging reporting and data system for ultrasound features of thyroid carcinoma. Thyroid. 2009;19(11):1257-64 Available at: http://www. liebertonline.com/doi/abs/10.1089/thy.2008.0021.

12. Kwak JY, Han KH, Yoon JH, Moon HJ, Son EJ, Park SH, et al. Thyroid imaging reporting and data system for US features of nodules: a step in establishing better stratification of Cancer risk. Radiology. 2011;260(3):892-9 Available at: http://pubs.rsna.org/doi/10.1148/radiol.11110206.

13. Tessler FN, Middleton WD, Grant EG, Hoang JK, Berland LL, Teefey SA, et al. ACR thyroid imaging, reporting and data system (TI-RADS): white paper of the ACR TI-RADS Committee. J Am Coll Radiol. 2017;14(5):587-95. https:// doi.org/10.1016/j.jacr.2017.01.046.

14. Moon HJ, Kwak JY, Kim E-K, Kim MJ. A taller-than-wide shape in thyroid nodules in transverse and longitudinal Ultrasonographic Planes and the prediction of malignancy. Thyroid. 2011;21(11):1249-53 Available at: http:// www.liebertonline.com/doi/abs/10.1089/thy.2010.0372.

15. Gul K, Ersoy R, Dirikoc A, Korukluoglu B, Ersoy PE, Aydin R, et al. Ultrasonographic evaluation of thyroid nodules: comparison of ultrasonographic, cytological, and histopathological findings. Endocrine. 2009;36(3):464-72.

16. Papini E, Guglielmi R, Bianchini A, Crescenzi A, Taccogna S, Nardi F, et al, Risk of malignancy in nonpalpable thyroid nodules: predictive value of ultrasound and color-doppler features. J Clin Endocrinol Metab. 2002;87(5): 1941-6.

17. Grant EG, Tessler FN, Hoang JK, Langer JE, Beland MD, Berland LL, et al. Thyroid ultrasound reporting lexicon: white paper of the ACR thyroid imaging, reporting and data system (TIRADS) committee. J Am Coll Radiol. 2015;12(12):1272-9. https://doi.org/10.1016/j.jacr.2015.07.011.
18. Middleton WD, Teefey SA, Reading CC, Langer JE, Beland MD, Szabunio MM et al. Comparison of performance characteristics of American College of Radiology TI-RADS, Korean Society of Thyroid Radiology TI-RADS, and American Thyroid Association guidelines. Am J Roentgenol. 2018;210(5): 1148-54.

19. Grani G, Lamartina L, Ascoli V, Bosco D, Nardi F, D'Ambrosio F, et al. Ultrasonography scoring systems can rule out malignancy in cytologically indeterminate thyroid nodules. Endocrine. 2017;57(2):256-61. https://doi. org/10.1007/s12020-016-1148-6.

20. Maia FFR, Matos PS, Pavin EJ, Zantut-Wittmann DE. Thyroid imaging reporting and data system score combined with Bethesda system for malignancy risk stratification in thyroid nodules with indeterminate results on cytology. Clin Endocrinol. 2015;82(3):439-44.

21. Trimboli P, Deandrea M, Mormile A, Ceriani L, Garino F, Limone PP, et al. American Thyroid Association ultrasound system for the initial assessment of thyroid nodules: use in stratifying the risk of malignancy of indeterminate lesions. Head Neck. 2018;40(4):722-7.

22. Trimboli P, Fulciniti F, Zilioli V, Ceriani L, Giovanella L. Accuracy of international ultrasound risk stratification Systems in Thyroid Lesions Cytologically Classified as indeterminate. Diagn Cytopathol. 2017:45(2):113-7.

23. Ulisse S, Bosco D, Nardi F, Nesca A, D'Armiento E, Guglielmino V, et al. Thyroid imaging reporting and data system score combined with the new Italian classification for thyroid cytology improves the clinical Management of Indeterminate Nodules. Int J Endocrinol. 2017;2017: 9692304.

24. Lee JH, Han K, Kim EK, Moon HJ, Yoon JH, Park WY, et al. Risk stratification of thyroid nodules with Atypia of undetermined significance/follicular lesion of undetermined significance (AUS/FLUS) cytology using ultrasonography patterns defined by the 2015 ATA guidelines. Ann Otol Rhinol Laryngol. 2017;126(9):625-33. https://doi.org/10.1177/0003489417719472.

25. Gao LY, Wang Y, Jiang YX, Yang X, Liu RY, Xi XH, et al. Ultrasound is helpful to differentiate Bethesda class III thyroid nodules: A PRISMA-compliant systematic review and meta-analysis. Med. 2017;96(16):e6564. Published online 2017 April 21. https://doi.org/10.1097/MD.0000000000006564.

26. Baser H, Cakir B, Topaloglu O, Alkan A, Polat SB, Dogan HT, et al. Diagnostic accuracy of thyroid imaging reporting and data system in the prediction of malignancy in nodules with atypia and follicular lesion of undetermined significance cytologies. Clin Endocrinol. 2017;86(4):584-90.

27. He YP, Xu HX, Zhao CK, Sun LP, Li XL, Yue WW, et al. Cytologically indeterminate thyroid nodules: increased diagnostic performance with combination of US TI-RADS and a new scoring system. Sci Rep. 2017;7(1):19. https://doi.org/10.1038/s41598-017-07353-y.

28. Baloch ZW, Fleisher S, LiVolsi VA, Gupta PK. Diagnosis of "follicular neoplasm": a gray zone in thyroid fine-needle aspiration cytology. Diagn Cytopathol. 2002;26(1):41-4.

29. Nikiforov YE, Carty SE, Chiosea SI, Coyne C, Duvvuri U, Ferris RL, et al. Highly accurate diagnosis of cancer in thyroid nodules with follicular neoplasm/ suspicious for a follicular neoplasm cytology by thyroseq V2 nextgeneration sequencing assay. Cancer. 2014;120(23):3627-34.

30. Labourier E, Shifrin A, Busseniers AE, Lupo MA, Manganelli ML, Andruss B, et al. Molecular testing for miRNA, mRNA, and DNA on fine-needle aspiration improves the preoperative diagnosis of thyroid nodules with indeterminate cytology. J Clin Endocrinol Metab. 2015;100(7):2743-50.

31. Yoon JH, Kwon HJ, Kim EK, Moon HJ, Kwak JY. Subcategorization of atypia of undetermined significance/follicular lesion of undetermined significance (AUS/FLUS): a study applying thyroid imaging reporting and data system (TIRADS). Clin Endocrinol. 2016:85(2):275-82.

32. Chng CL, Kurzawinski TR, Beale T. Value of sonographic features in predicting malignancy in thyroid nodules diagnosed as follicular neoplasm on cytology. Clin Endocrinol. 2015;83(5):711-6.

33. Rocha TG, Rosario PW, Silva AL, Nunes MB, Silva TH, Oliveira PHL, et al. Ultrasonography classification of the American Thyroid Association for predicting malignancy in thyroid nodules $>1 \mathrm{~cm}$ with indeterminate cytology : a prospective study authors. Horm Metab Res. 2018;50:597-601.

34. Tang AL, Falciglia M, Yang H, Mark JR, Steward DL. Validation of American Thyroid Association ultrasound risk assessment of thyroid nodules selected for ultrasound fine-needle aspiration. Thyroid. 2017;27(8):1077-82 Available at: http://online.liebertpub.com/doi/10.1089/thy.2016.0555.

35. Bongiovanni M, Spitale A, Faquin WC, Mazzucchelli L, Baloch ZW. The Bethesda system for reporting thyroid cytopathology: a meta-analysis. Acta Cytol. 2012;56(4):333-9. 
36. Chaigneau E, Russ G, Royer B, Bigorgne C, Bienvenu-Perrard M, Rouxel A, et al. TIRADS score is of limited clinical value for risk stratification of indeterminate cytological results. Eur J Endocrinol. 2018;179(1):13-20.

37. Valderrabano P, Mcgettigan MJ, Lam CA. Thyroid nodules with indeterminate cytology : utility of the American Thyroid Association sonographic patterns for cancer risk. Thyroid. 2018;28(8):1-29.

38. Persichetti A, Di Stasio E, Guglielmi R, Bizzarri G, Taccogna S, Misischi I, et al. Predictive value of malignancy of thyroid nodule ultrasound classification systems: a prospective study. J Clin Endocrinol Metab Clin Endocrinol Metab. 2018;103(April):1359-68.

39. Yoon JH, Lee HS, Kim E-K, Moon HJ, Kwak JY. Malignancy risk stratification of thyroid nodules: comparison between the thyroid imaging reporting and data system and the 2014 American Thyroid Association management guidelines. Radiology. 2016;278(3):917-24 Available at: http://pubs.rsna.org/ doi/10.1148/radiol.2015150056.

40. Horvath E, Silva CF, Majilis S, Rodriguez I, Skoknic V, Castro A, et al. Prospective validation of the ultrasound based TIRADS (thyroid imaging reporting and data system) classification: results in surgically resected thyroid nodules. Eur Radiol. 2017;27(6):2619-28.

41. Russ G, Bonnema SJ, Erdogan MF, Durante C, Ngu R, Leenhardt L. European Thyroid Association Guidelines for Ultrasound Malignancy Risk Stratification of Thyroid Nodules in Adults: The EU-TIRADS. Eur Thyroid J. 2017:225-37 Available at: http://www.karger.com/?doi=10.1159/000478927.

42. Zhang J, Liu BJ, Xu HX, Xu JM, Zhang YF, Liu C, et al. Prospective validation of an ultrasound-based thyroid imaging reporting and data system (TIRADS) on 3980 thyroid nodules. Int J Clin Exp Med. 2015;8(4):5911-7.

43. de Koster EJ, de Geus-Oei L-F, Dekkers OM, van Engen-van Grunsven I, Hamming J, Corssmit EPM, et al. Diagnostic utility of molecular and imaging biomarkers in cytological indeterminate thyroid nodules. Endocr Rev. 2018; 39(2):154-91 Available at: http://academic.oup.com/edrv/advance-article/ doi/10.1210/er.2017-00133/4782710.

\section{Publisher's Note}

Springer Nature remains neutral with regard to jurisdictional claims in published maps and institutional affiliations.

Ready to submit your research? Choose BMC and benefit from:

- fast, convenient online submission

- thorough peer review by experienced researchers in your field

- rapid publication on acceptance

- support for research data, including large and complex data types

- gold Open Access which fosters wider collaboration and increased citations

- maximum visibility for your research: over $100 \mathrm{M}$ website views per year

At $\mathrm{BMC}$, research is always in progress.

Learn more biomedcentral.com/submissions 